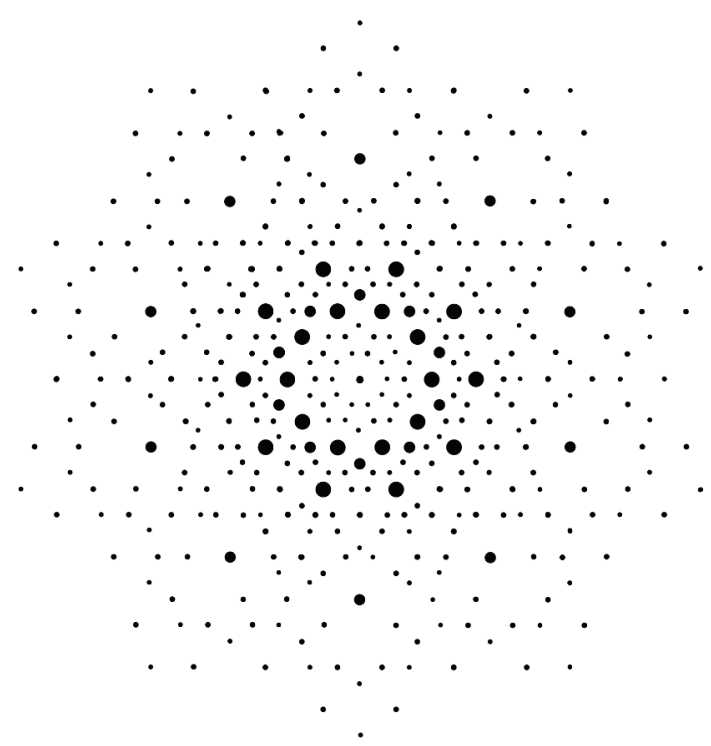

Figure 1. The simulated diffraction pattern along a quasicrystalline five-fold axis, composed of contributions from the multiply twinned golden rhombohedra and completed by dynamical scattering (the smallest radii).

Keywords: quasicrystals, unit-cell twinning, long-range order
MS30. Structure and function

in coordination compounds
Chairs: Marijana Đaković, Janusz Lipkowski

\section{MS30-01 Hybrid porous materials}

Michael J. Zaworotko ${ }^{1}$

1. Department of Chemical and Environmental Science, University of Limerick, Ireland

email:xtal@ul.ie

That composition and structure profoundly impact the properties of crystalline solids has provided impetus for exponential growth in the field of crystal engineering [1] over the past 25 years. This lecture will address how crystal engineering has evolved from structure design (form) to control over bulk properties (function) with particular emphasis upon an underexplored class of porous material: hybrid organic-inorganic compounds. Whereas porous crystalline materials such as purely inorganic materials (e.g. zeolites) and those based upon coordination chemistry (e.g. Metal-Organic Frameworks and Porous Coordination Polymers) are well studied and offer great promise for separations and catalysis, they can be handicapped by cost or performance (e.g. chemical stability, interference from water or low selectivity) limitations. Hybrid Porous Materials, HPMs, are much less studied. HPMs are built from metal or metal cluster "nodes" and combinations of organic and inorganic "linkers" and they represent an opportunity to overcome the weaknesses associated with existing classes of porous material. Two prototypal families of HPMs will be addressed: (i) Pillared square grids with pcu topology can afford exceptional control over pore chemistry, pore size and binding energy for CO2.[2] Further, their performance is typically unaffected by moisture. (ii) $\mathrm{mmo}$ nets are based upon square grids linked by angular inorganic linkers such as chromate anions.[3] They also offer exceptional performance with respect to capture of $\mathrm{CO} 2$ and other polarizable gases. New results related to the structure and properties of pcu and mmo HPMs will be presented. 1. (a) Desiraju, G.R. Crystal engineering: The design of organic solids Elsevier, 1989; (b) Moulton, B.; Zaworotko, M.J. Chemical Reviews 2001, 101, 1629-1658. 2. (a) Burd, S.D.; Ma, S.; Perman, J.A.; Sikora, B.J.; Snurr, R.Q.; Thallapally, P.K.; Tian, J.; Wojtas, L.; Zaworotko, M.J. J. Amer. Chem. Soc. 2012, 134, 3663-3666. (b) Nugent, P.; Belmabkhout, Y.; Burd, S.D.; Cairns, A.J.; Luebke, R.; Forrest, K.; Pham, T.; Ma, S.; Space, B.; Wojtas, L.; Eddaoudi, M.; Zaworotko, M.J. Nature 2013, 495, 80-84, 2013. 3. Mohamed, M.; Elsaidi, S.; Wojtas, L.; Pham, T.; Forrest, K.A.; Tudor, B.; Space, B.; Zaworotko, M.J. J. Amer. Chem. Soc. 2012, 134, 19556-19559.

Keywords: crystal engineering, porous materials, carbon capture 